\title{
The Role of Kidney-Pancreas Transplantation in Diabetic Kidney Disease
}

\author{
Alexander C. Wiseman \\ Published online: 27 July 2010 \\ (C) The Author(s) 2010. This article is published with open access at Springerlink.com
}

\begin{abstract}
For patients with type 1 diabetes, innovations in insulin formulations and delivery have improved the ability to achieve excellent blood glucose control. However, it is uncommon to achieve euglycemia, particularly while avoiding complications arising from hypoglycemia. Pancreas transplantation remains the only broadly applied treatment strategy that can result in normalization of blood glucose, but this must be weighed against the risks of a surgical procedure and subsequent immunosuppression. To improve this risk/benefit ratio, pancreas transplantation is typically performed in patients with kidney failure who are to undergo kidney transplantation and immunosuppression (simultaneous pancreas-kidney transplant) or who have undergone kidney transplant and are obligated to the use of immunosuppressive medications (pancreas after kidney transplant). The purpose of this review is to clarify the benefit of an added pancreas transplant in these clinical settings and formulate an approach to the patient with type 1 diabetes as they approach kidney failure.
\end{abstract}

Keywords Pancreas transplantation. Type 1 diabetes . Chronic kidney disease

\section{Introduction}

Significant improvements in the management of type 1 diabetes mellitus (T1DM) have resulted in a decrease in the projected incidence of kidney failure over time. Once considered to impact $15 \%$ of patients who survive with

A. C. Wiseman $(\square)$

Transplant Center, University of Colorado Denver,

Mail Stop F749, AOP 7089, 1635 North Aurora Court,

Aurora, CO 80045, USA

e-mail: Alexander.wiseman@ucdenver.edu diabetes for 30 years [1], a recent epidemiologic study suggests that the incidence of kidney failure may now be less than $8 \%$ [2]. The benefits of intensive blood glucose control on the progression of kidney disease have been well documented; the DCCT (Diabetes Control and Complications Trial) and EDIC (Epidemiology of Diabetes Interventions and Complications) cohort of 1349 subjects reported a total of only 11 cases of end-stage renal disease over a mean duration of diabetes of nearly 20 years, with a mean hemoglobin $\mathrm{A}_{1 \mathrm{c}}$ of $7.2 \%$ and $8.0 \%$ in the 6.5 and 8 years of consecutive follow-up that these trials provided [3, 4].

Unfortunately, many patients cannot achieve the degree of blood glucose control that is reported in the setting of a clinical trial, or suffer untoward effects of hypoglycemia with aggressive control that can threaten quality of life and be lifethreatening. There remains a need for additional therapeutic options for these patients. Pancreas transplantation can offer the benefits of normalization of blood glucose control but at the risk of a surgical procedure and the requirement of antirejection medications to sustain pancreas function. Fortunately, advances in surgical outcomes and immunosuppression over the past decade have made pancreas transplantation a more attractive option for patients with T1DM and kidney failure, and have led to a reconsideration of the most appropriate timing of the procedure in relation to an individual's kidney function (either as a simultaneous pancreas-kidney transplant [SPK], a pancreas after kidney transplant $[\mathrm{PAK}]$, or pancreas transplant alone [PTA]).

\section{The Critical Role of Kidney Transplantation in Patients with T1DM}

For patients with T1DM who suffer from progressive kidney disease, it is imperative that proper education 
regarding options for renal replacement therapy is discussed proactively due to the significant negative impact dialysis imparts upon patient survival, and the dramatic benefits that can be achieved with kidney transplantation. For patients with diabetes who are placed on a waiting list for kidney transplantation but remain on dialysis, the mean survival is 8 years, whereas for those who receive a kidney the mean survival is 22 years [5]. Similarly, for those who are eligible for a combined kidney pancreas transplant (likely a slightly healthier population due to acceptance criteria), patients who remain on dialysis have an estimated mortality of $40 \%$ at 4 years, whereas those who receive SPK transplants have a mortality of $10 \%$ [6]. The timing of kidney transplantation is also a critical determinant to outcomes. If performed before the need for dialysis (preemptive transplant), kidney transplants from a living donor result in a $43 \%$ reduction in risk of death compared with transplant after the onset of dialysis, whereas kidney transplants from a deceased donor are associated with a $12 \%$ reduction in risk of death [7]. Given these dramatic differences in outcomes, patients with T1DM and estimated glomerular filtration rate (GFR) of $30 \mathrm{~mL} / \mathrm{min}$ should be referred to a transplant center for pretransplant evaluation and counseling regarding potential transplant options.

\section{The Impact of Pancreas Transplantation in Kidney Transplant Candidates and Recipients}

For an individual patient with T1DM and progressive renal disease, transplant options include kidney transplant alone (from a deceased or living donor [DDKA, LDKA]) or SPK. The benefits of adding a pancreas to a kidney transplant should be considered in terms of "hard" end points (ie, the impact on patient and kidney graft survival) and "softer" end points (ie, the benefits of euglycemia on quality of life and other secondary complications of diabetes such as the impact on neuropathy, retinopathy, and cardiovascular disease).

\section{Pancreas Transplantation and Patient Survival, Kidney Graft Survival}

Regarding patient survival, it is clear from numerous retrospective analyses and data registries that an SPK procedure is associated with a higher postoperative mortality of about $2 \%$ in the first 90 days compared with kidney alone (KA) $[8,9 \bullet]$. This difference is mitigated by about 12 to 24 months when compared with DDKA, and patient survival is markedly improved thereafter for SPK. This is likely due not only to the euglycemia that is provided by the pancreas transplant, but also is related to differences in deceased donor characteristics of an SPK donor versus a
KA donor $[10 \bullet, 11 \bullet]$. Only about $15 \%$ to $20 \%$ of deceased organ donors are suitable for both pancreas and kidney donation $[12,13]$, and these donors are younger, with lower body mass index (BMI) and less comorbidities prior to brain death than donors who are not pancreas donors [14]. When comparing kidney graft survival in SPK recipients and DDKA recipients, again the SPK provides better outcomes than DDKA, beginning as early as 12 months after the procedure.

The benefits of SPK over LDKA are more subtle than those noted compared with DDKA, primarily due to a significantly lower risk of death in the postoperative period with the LDKA procedure [15•]. With LDKA, recipients are known to be clinically stable at the time of surgery rather than "on call" for potential transplant, the donor is extensively evaluated, the surgery is done on a scheduled basis with a minimum of time from kidney removal to transplantation ("cold ischemia time"), and is less complex than an SPK procedure, all of which lead to fewer postoperative complications and immediate graft function. For these reasons, 1-year mortality for SPK versus LDKA is $5 \%$, versus $2 \%$ to $3 \%$, from various reports $[9 \bullet, 15 \bullet]$.

Three important recent publications have addressed the value of SPK versus LDKA and DDKA in retrospective analyses using three separate transplant registries. Using the Organ Procurement and Transplantation Network/United Network of Organ Sharing database (comprised of kidney transplant and pancreas transplant recipients in the United States), Young et al. [16••] compared kidney graft and patient survival differences with SPK, LDKA, and DDKA over the period 2000 to 2007. One-year survival was highest in the LDKA cohort and lowest in the DDKA cohort $(95 \%, 97 \%$, and $93 \%$, respectively), as was kidney graft survival $(93 \%, 95 \%$, and $89 \%$, respectively). These differences persisted for 5 to 6 years, following which time the outcomes of SPK and LDKA began to shift in favor of SPK. At 6 years, patient survival was higher in the SPK cohort with similar kidney graft survival to LDKA (patient survival $85 \%, 80 \%$, and $69 \%$, and graft survival $72 \%, 72 \%$, $58 \%$, respectively). Although Young et al. [16••] concluded that LDKA should be pursued whenever possible due to unpredictable organ availability and at least equivalent if not better outcomes with LDKA than SPK over a 5- to 6year period, these data suggested that patients with T1DM may derive greater benefits in the long term from SPK. Morath et al. [17••] clarified this point using data from the Collaborative Transplant Study (comprised of transplant centers in the United States and internationally). In patients with kidney function at 10 years post-transplant, those who had received SPK had a significantly better survival rate over the following 8 years than patients who had received an LDKA or DDKA. This improved survival was noted particularly in those patients with functioning pancreas 
grafts at 10 years and was associated with less cardiovascular death in particular, leading the authors to conclude that longterm glycemic control in SPK recipients conferred a survival benefit over LDKA, due to less progression of cardiovascular disease. A follow-up analysis from the same group suggested that the benefit of a pancreas transplant outweighs the early risk of the surgical procedure at about 9 to 10 years after transplant irrespective of pancreas function [18].

This "delayed gratification" for a survival benefit with SPK poses questions regarding if this is an appropriate alternative to LDKA when a living kidney donor is available. This is particularly problematic when one considers that the median pancreas life span is slightly greater than 10 years, and that $15 \%$ of SPK recipients lose pancreas graft function within the first year [15•]. To better understand the hazards of early pancreas graft loss and the benefits of early pancreas graft function on long-term survival, Weiss et al. [19••] compared patients considered healthy enough to be SPK candidates but received LDKA and DDKA to patients who received SPK. This analysis examined survivors at 12 months who had kidney function, and segregated the SPK cohort into those who had pancreas function at 12 months (SPK,P+) versus those with loss of function (SPK,P-). The SPK $(\mathrm{P}+)$ cohort had significantly better estimated survival throughout the following 7 years $(88.6 \%)$ than the remaining three groups, including the LDKA group (80.0\%), which had a nonstatistically better survival advantage over those SPK recipients who had lost function in the first year (SPK,P-; 73.9\%). This point was reiterated in a large single-center analysis that demonstrated that in the $12 \%$ of SPK recipients who had early loss of pancreas function (within 90 days), patient and kidney graft survival were significantly worse than SPK recipients with early function of the pancreas [20].
Based on the findings of these three analyses, the following conclusions can be drawn: 1) DDKA is inferior to LDKA or SPK for both patient and kidney graft survival; 2 ) the increased mortality of about $2 \%$ and pancreas graft loss of $10 \%$ to $15 \%$ for SPK in the first year leads to slightly better LDKA patient survival for a least 6 years; 3 ) SPK ultimately leads to improved patient survival, and is dependent on pancreas function; 4) with present-day outcomes, it can be expected that about $85 \%$ of SPK recipients will derive greater survival benefit from SPK versus LDKA, which can be evident as early as 12 months post-transplant (Table 1).

\section{Pancreas Transplantation and Secondary Complications of Diabetes}

Unfortunately, few recent trials have been performed that examine the effect of normalization of blood glucose with pancreas transplant on complications such as retinopathy, neuropathy, and nephropathy. Further, these trials often are small and without adequate control groups, or use surrogate markers of improvement that may not reflect improvements that may be noted clinically. Highlights of these trials will be briefly reviewed as they are often used as criteria to consider PTA in patients with early renal disease.

The prevention of diabetic nephropathy (DN) is often raised as an indication for PTA, prior to severe kidney failure. The most widely accepted study addressing the potential for stabilization or reversal of DN is a case series of eight PTA recipients who maintained pancreas graft function for 10 years, who underwent assessment and kidney biopsy at 0,5 , and 10 years post-PTA [21]. Microalbuminuria resolved, and the early histologic find-

Table 1 Summary of advantages and disadvantages of transplant options for diabetic kidney disease

\begin{tabular}{|c|c|c|}
\hline & Advantages & Disadvantages \\
\hline DDKA & Provides better survival than dialysis options & $\begin{array}{l}\text { Inferior to other transplant options with respect to } \\
\text { kidney graft survival and patient survival }\end{array}$ \\
\hline \multirow[t]{2}{*}{ LDKA } & Minimizes waiting time, time spent on dialysis & No normalization of blood glucose \\
\hline & $\begin{array}{l}\text { Very low early morbidity and mortality } \\
\text { Better survival initially than with SPK }\end{array}$ & $\begin{array}{l}\text { Inferior patient survival over time when compared } \\
\text { with SPK recipients with functioning grafts }\end{array}$ \\
\hline \multirow[t]{2}{*}{ SPK } & $\begin{array}{l}\text { Glycemic control, with recent median pancreas } \\
\text { graft survival of }>10 \text { years }\end{array}$ & Higher morbidity and mortality due to larger operation \\
\hline & High-quality, deceased donor kidney transplant. & $\begin{array}{l}\text { If pancreas fails within the first year, outcomes are } \\
\text { worse than LDKA }\end{array}$ \\
\hline \multirow[t]{2}{*}{ PAK } & Glycemic control & $\begin{array}{l}\text { Two separate surgical procedures, increased mortality } \\
\text { early postoperatively following pancreas transplant }\end{array}$ \\
\hline & $\begin{array}{l}\text { If living donor kidney transplant, comparable/better } \\
\text { patient and kidney graft survival than LDKA }\end{array}$ & $\begin{array}{l}\text { Historically inferior pancreas graft survival ( } 35 \% \text { at } \\
10 \text { years) than SPK }\end{array}$ \\
\hline
\end{tabular}

$D D K A$ deceased donor kidney alone, $L D K A$ living donor kidney alone, $P A K$ pancreas after kidney transplant, $S P K$ simultaneous pancreas-kidney transplant 
ings of DN stabilized and regressed, but this was not noted until the 10-year biopsy. Further, estimated GFR fell 30\% early in the study, presumably due to the vasoconstrictive and nephrotoxic immunosuppressive agent cyclosporine. Current recommendations for immunosuppression for all types of pancreas transplants (SPK, PAK, and PTA) all include a calcineurin inhibitor such as tacrolimus or cyclosporine. Given this trade-off in GFR for stabilization of DN, PTA should not be recommended solely for prevention of DN. Diabetic retinopathy may improve, stabilize, or deteriorate following the pancreas transplantation [22-24]. One recent study compared 33 patients following PTA with a control group of 35 patients with similar baseline degree of retinopathy. Over a mean follow-up of 2.5 years, $91 \%$ of patients improved or stabilized in the PTA cohort versus $43 \%$ in the control group $(P<0.01)$ [22]. Finally, early studies using surrogate markers (electrophysiologic measures) of diabetic neuropathy have suggested a beneficial impact on neuropathy, with improvements in gastric emptying and sensory and motor neuropathy $[25,26]$. Aside from improvements in patient and kidney graft survival, perhaps the most valuable benefit achieved with pancreas transplantation is the avoidance of hypoglycemia unawareness and improvement in diabetes-related quality of life [27, 28]. Hyperinsulinemic clamp studies in PTA recipients demonstrate improvement in glucagon and epinephrine responses and symptom recognition that persisted up to 19 years after PTA [29]. In total, these studies suggest that pancreas transplant may result in improvements in early complications of diabetes and result in improved diabetesrelated quality of life, but may not be as successful in more advanced disease [30], and further controlled studies are needed.

\section{SPK Transplantation or PAK Transplantation?}

For the patient who selects the option of LDKA rather than wait for an SPK (either due to the increased mortality risk noted above or an unacceptably long waiting time for an appropriate deceased donor), another question emerges: what are the risks and benefits of an added pancreas transplant following kidney transplant (PAK)? Unlike the comparison of SPK versus remaining on dialysis, in which the mortality while accumulating time on dialysis is significant and leads to a clear advantage for SPK, it is difficult to overcome the risks of pancreas transplant surgery and demonstrate a benefit when comparing PAK versus LDKA due to the low mortality rates of LDKA recipients. This is best demonstrated by comparing two studies that examined PAK outcomes from registry data.
From PAK performed in 1995 to 2000, Venstrom et al. [31] showed a nearly threefold increased risk of death in the 90 days following PAK compared with "conventional therapy" following kidney transplant alone, and although this risk dissipated over time, PAK was associated with a $42 \%$ increased risk of death at 4 years following transplant. Gruessner et al. [6] performed a similar analysis of PAK recipients from 1995 to 2003 . They also showed a greater than fourfold increased risk of death in the first 90 days, but unlike Venstrom et al. [31], this risk was entirely mitigated due to a reduction in risk after 1 year (hazard ratio for death, 0.18; PAK vs conventional therapy, $P<0.001$ ), and there was no increased risk of death overall. Differences in study design between these reports that account for these different conclusions include exclusion of patients with a creatinine greater than 2.0 in the report by Venstrom et al. [31] (thus creating a healthier waiting list for comparison) and the longer study period ( 9 vs 5 years) by Gruessner et al. [6] (thus permitting the benefits of glycemic control to emerge). Overall, these findings suggest that the mortality risk of an added pancreas surgery after kidney transplant is not overcome at 4 years but that PAK perhaps is advantageous with longer follow-up.

Several recent reports have shed a more favorable light on PAK transplant following LDKA (pancreas after living kidney transplant [PALK]). In a large registry analysis comparing patients receiving LDKA with those who received a PALK during the period 1997 to 2007, 8-year estimated patient ( $85 \%$ vs $75 \%$ ) and kidney graft survival ( $75 \%$ vs $62 \%)$ were superior in the PALK group, with advantages that persisted after correction for other comorbidities and when examining patients with kidney function at 1 year following LDKA [32•]. A single-center report compared 175 patients who underwent PALK with 75 patients who received LDKA and were eligible for PAK but did not receive a pancreas transplant due to financial or personal reasons [33]. Patient survival and kidney graft survival were equivalent at 1,5 , and 10 years, better hemoglobin $\mathrm{A}_{1 \mathrm{c}}$ was achieved in PALK versus LDKA, and better GFR over time was noted in those PALK who had a pancreas transplant 2 to 12 months following kidney transplant. These reports suggest that the mortality risk of pancreas transplant may be minimized in a more modern era and in experienced pancreas transplant centers, favoring PALK over LDKA.

These improvements in PALK outcomes occurred despite the fact that pancreas graft function is considerably shorter in the setting of PAK than SPK. From 2004 to 2008, the1- and 3-year pancreas function rates for SPK were 85\% and $78 \%$, whereas for PAK these rates were $79 \%$ and $65 \%$, respectively [9॰]. These differences appear to be due to a higher rate of acute and chronic rejection in the setting of PAK (at 3 years, graft loss due to immunologic causes was 
$14 \%$ in PAK vs $5 \%$ in SPK), presumably because of a separate antigenic stimulus in PAK and difficulty in monitoring for isolated pancreas rejection. Single centers have recently reported novel strategies of immunosuppression, primarily relying upon depleting T-cell induction therapy, that have been associated with improvements in pancreas graft function in the setting of PAK [34•]. These improvements have led to the suggestion that PALK may be a preferred strategy over SPK. This recommendation must be balanced with the recognition that PALK recipients are a selected group of patients who have had favorable initial kidney transplant outcomes and are considered candidates for an additional immunosuppression and a second operation (Table 1).

\section{Opportunities for Growth of Pancreas Transplantation}

Ultimately, the decision for a patient to receive an SPK or receive a living donor kidney and consider a pancreas transplant at a later date is dependent upon the waiting time necessary to receive an SPK transplant. In the United States there are significant regional variations in waiting time for an SPK transplant. Some regions have a policy that all patients awaiting SPK are combined with the kidney transplant waiting list, whereas others have a separate SPK list. In the former, waiting times for SPK are as long as for kidney transplant alone, generally 4 to 5 years, whereas in the latter, waiting time is significantly less, often less than 1 year. Efforts to standardize the SPK waiting list policy nationally are underway in patients with T1DM. Because patients who receive an SPK enjoy the greatest survival benefit of all transplanted patients compared with dialysis yet comprise only $4 \%$ of the kidney transplant waiting list [35], a proposal to ensure that a separate SPK waiting list with priority over kidney transplantation alone has gained significant support.

Although efforts to prioritize SPK organ allocation will lead to improvements in the utility of organs transplanted (by providing a greater lifetime benefit of kidney transplants overall), this will not necessarily lead to a greater number of SPK transplants performed overall. The pancreas is successfully transplanted from only $15 \%$ to $20 \%$ of potential deceased organ donors, primarily due to perceived organ quality by transplant centers. To understand reasons for refusal of pancreata from deceased donors who appear otherwise optimal for solid organ donation, we performed an analysis of donor characteristics of successful pancreas donors and compared these donors with a groups of deceased donors 19 to 40 years of age whose liver and kidneys were successfully transplanted [36]. This study revealed that subjective assessments of organ quality formed the basis for most unused donor pancreata, and the potential for growth in pancreas transplant required better standardization
Fig. 1 Therapeutic approach to the patient with type 1 diabetes mellitus (T1DM) and progressive kidney failure (glomerular filtration rate $[\mathrm{GFR}]<30 \mathrm{~mL} / \mathrm{min}$ )

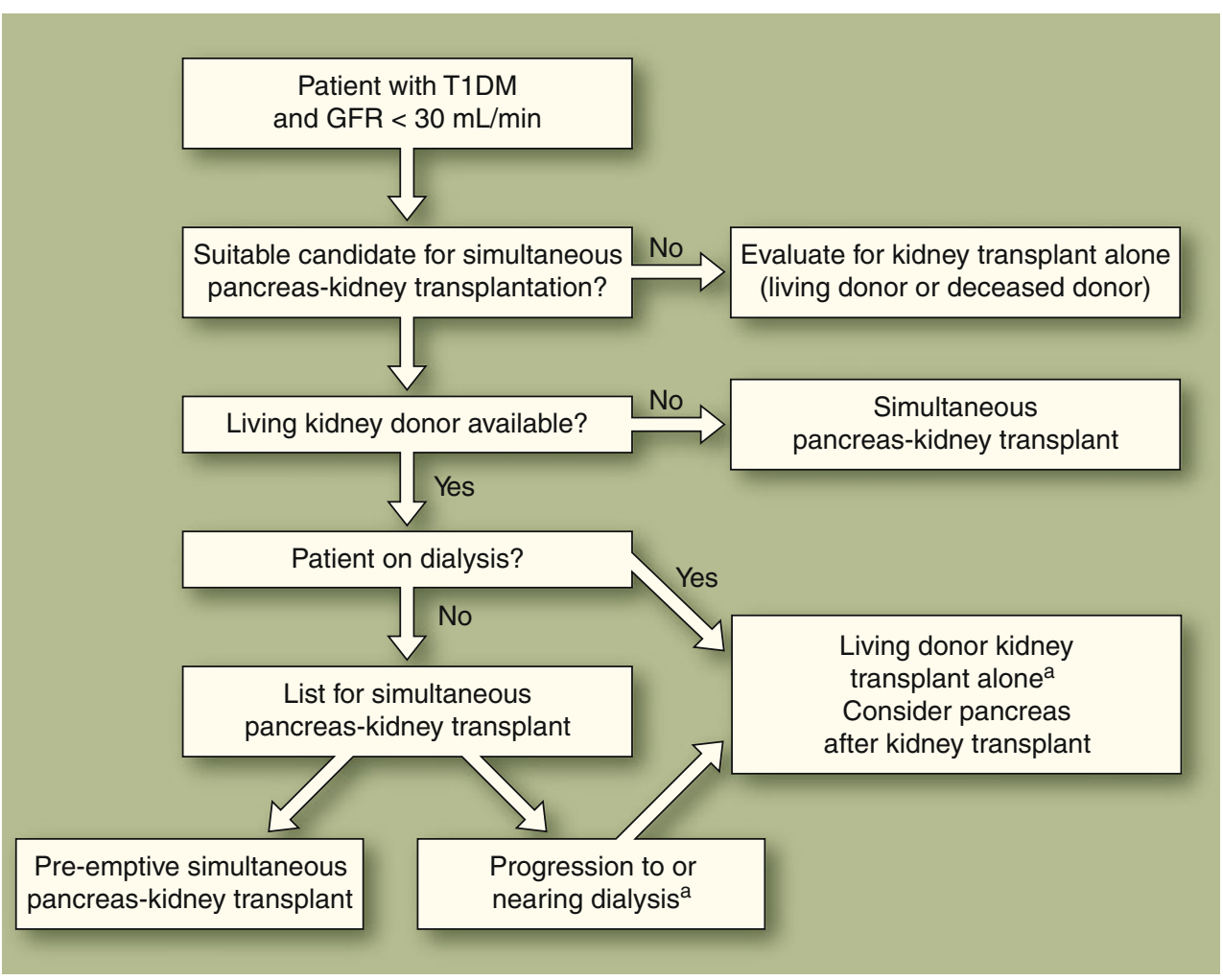


of organ and donor quality. Recently, a pancreas "donor risk index" that uses 10 donor characteristics (including age, BMI, cause of death, and donor renal function) was demonstrated to predict pancreas graft function [37]. Ideally, such objective assessments may lead to a greater utilization rate of pancreata from deceased donors.

\section{Evidence-based Recommendations}

Given these recent advances, recommendations for patients with T1DM approaching kidney failure should be tailored to the individual's circumstance, and should include an assessment of the following: 1) Can a living donor can be identified? 2) Is the patient (and transplant program) willing to accept a higher risk of death and possibility of pancreas graft loss ( $\sim 2 \%$ and $\sim 15 \%$ in the first year, respectively) when considering SPK? 3) How debilitating are the patient's diabetes-related quality-of-life issues and achieved level of glycemic control? and 4) What is the expected waiting time for an SPK in the patient's geographic region? In general, SPK appears to offer advantages over KA (either DDKA or LDKA) if the waiting time for a deceased donor is not excessive, and dialysis time can be minimized (perhaps to $<6$ months). For those patients who are unable to wait for SPK, living donor kidney transplant followed by a later pancreas transplant (PALK) appears to be associated with better kidney graft function, with a risk of mortality that is similar to LDKA (Fig. 1).

\section{Conclusions}

Pancreas transplantation can improve patient survival, kidney graft function, and quality of life in patients with T1DM and kidney disease. Recent improvements in surgical outcomes and immunosuppression have translated to improvements in graft function in both the SPK and PAK setting, and national organ allocation policy is now recognizing the need for emphasis of pancreas transplantation. With newer objective tools to assess the adequacy of deceased donors for pancreas transplantation, it is hoped that pancreas transplantation can expand and benefit a substantially greater number of patients in the future.

Disclosure No potential conflict of interest relevant to this article was reported.

Open Access This article is distributed under the terms of the Creative Commons Attribution Noncommercial License which permits any noncommercial use, distribution, and reproduction in any medium, provided the original author(s) and source are credited.

\section{References}

Papers of particular interest, published recently, have been highlighted as:

- Of importance

•• Of major importance

1. Krolewski M, Eggers PW, Warram JH: Magnitude of end-stage renal disease in IDDM: a 35 year follow-up study. Kidney Int 1996, 50:2041-2046.

2. Finne $P$, Reunanen $A$, Stenman $S$, et al.: Incidence of end-stage renal disease in patients with type 1 diabetes. JAMA 2005, 294:1782-1787.

3. The effect of intensive treatment of diabetes on the development and progression of long-term complications in insulin-dependent diabetes mellitus. The Diabetes Control and Complications Trial Research Group [no authors listed]. N Engl J Med 1993, 329: 977-986.

4. Writing Team for the Diabetes Control and Complications Trial/ Epidemiology of Diabetes Interventions and Complications Research Group: Sustained effect of intensive treatment of type 1 diabetes mellitus on development and progression of diabetic nephropathy: the Epidemiology of Diabetes Interventions and Complications (EDIC) study. JAMA 2003, 290:2159-2167.

5. Wolfe RA, Ashby VB, Milford EL, et al.: Comparison of mortality in all patients on dialysis, patients on dialysis awaiting transplantation, and recipients of a first cadaveric transplant. $\mathrm{N}$ Engl J Med 1999, 341:1725-1730.

6. Gruessner RW, Sutherland DE, Gruessner AC: Mortality assessment for pancreas transplants. Am J Transplant 2004, 4:2018-2026.

7. Becker BN, Rush SH, Dykstra DM, et al.: Preemptive transplantation for patients with diabetes-related kidney disease. Arch Intern Med 2006, 166:44-48.

8. Israni AK, Feldman HI, Propert KJ, et al.: Impact of simultaneous kidney-pancreas transplant and timing of transplant on kidney allograft survival. Am J Transplant 2005, 5:374-382.

9. - Gruessner AC, Sutherland DE, Gruessner RW: Pancreas transplantation in the United States: a review. Curr Opin Organ Transplant 2010, 15: 93-101. This is an excellent resource document that summarizes pancreas transplant outcomes in the most recent era from 2004 to 2008.

10. - Weiss AS, Smits G, Wiseman AC: Standard criteria donor pancreas donation status is associated with improved kidney transplant outcomes. Clin Transplant 2009, 23:732-739. This article defines characteristics of deceased organ donors that are associated with enhanced kidney graft survival. It describes the added benefit that donors who are both pancreas and kidney donors confer upon recipients.

11. - Weiss AS, Smits G, Wiseman AC: Simultaneous pancreaskidney versus deceased donor kidney transplant: can a fair comparison be made? Transplantation 2009, 87:1402-1410. This article defines characteristics of deceased organ donors that are associated with enhanced kidney graft survival. It reports that kidneys transplanted from donors who are both kidney and pancreas donors provide 5-year outcomes equivalent to simultaneous kidney pancreas transplants in patients with TIDM.

12. Tuttle-Newhall JE, Krishnan SM, Levy MF, et al.: Organ donation and utilization in the United States: 1998-2007. Am J Transplant 2009, 9(4 Pt 2):879-893.

13. Wolfe RA, Merion RM, Roys EC, Port FK: Trends in organ donation and transplantation in the United States, 1998-2007. Am J Transplant 2009, 9(4 Pt 2):869-878.

14. Stegall MD, Dean PG, Sung R, et al.: The rationale for the new deceased donor pancreas allocation schema. Transplantation 2007, 83:1156-1161. 
15. - McCullough KP, Keith DS, Meyer KH, et al.: Kidney and pancreas transplantation in the United States, 1998-2007: access for patients with diabetes and end-stage renal disease. Am J Transplant 2009, 9(4 Pt 2):894-906. This is an outstanding summary of outcomes of kidney and pancreas transplantation and a discussion of trends in the waiting list for SPK and PAK.

16. •• Young BY, Gill J, Huang E, et al.: Living donor kidney versus simultaneous pancreas-kidney transplant in type I diabetics: an analysis of the OPTN/UNOS database. Clin J Am Soc Nephrol 2009, 4:845-852. This report provides an argument in support of living donor kidney transplantation rather than simultaneous kidney transplantation, with counterpoints to this argument provided in references $[17 \cdot \bullet]$ and $[19 \cdot \bullet]$.

17. •• Morath C, Zeier M, Döhler B, et al.: Metabolic control improves long-term renal allograft and patient survival in type 1 diabetes. J Am Soc Nephrol 2008, 19:1557-1563. This articles discusses the long-term data in support of the premise that the long-term benefits of pancreas transplantation outweigh the early risks in patients with TIDM and kidney failure.

18. Morath C, Zeier M, Döhler B, et al.: Transplantation of the type 1 diabetic patient: the long-term benefit of a functioning pancreas allograft. Clin J Am Soc Nephrol 2010, 5:549-552.

19. •• Weiss AS, Smits G, Wiseman AC: Twelve-month pancreas graft function significantly influences survival following simultaneous pancreas-kidney transplantation. Clin J Am Soc Nephrol 2009, 4:988-995. Evidence is provided that as little as 12 months of pancreas transplant function can lead to significantly improved patient survival, and that SPK can be considered the treatment of choice for patients with TIDM approaching the need for kidney transplantation.

20. Hill M, Garcia R, Dunn T, et al.: What happens to the kidney in an SPK transplant when the pancreas fails due to a technical complication? Clin Transplant 2008, 22:456-461.

21. Fioretto P, Steffes MW, Sutherland DE, et al.: Reversal of lesions of diabetic nephropathy after pancreas transplantation. N Engl J Med 1998, 339:69-75.

22. Giannarelli R, Coppelli A, Sartini MS, et al.: Pancreas transplant alone has beneficial effects on retinopathy in type 1 diabetic patients. Diabetologia 2006, 49:2977-2982.

23. Pearce IA, Ilango B, Sells RA, Wong D: Stabilisation of diabetic retinopathy following simultaneous pancreas and kidney transplant. Br J Ophthalmol 2000, 84:736-740.

24. Ramsay RC, Goetz FC, Sutherland DE, et al.: Progression of diabetic retinopathy after pancreas transplantation for insulindependent diabetes mellitus. N Engl J Med 1988, 318:208-214.
25. Martinenghi S, Comi G, Galardi G, et al.: Amelioration of nerve conduction velocity following simultaneous kidney/pancreas transplantation is due to the glycaemic control provided by the pancreas. Diabetologia 1997, 40:1110-1112.

26. Navarro X, Sutherland DE, Kennedy WR: Long-term effects of pancreatic transplantation on diabetic neuropathy. Ann Neurol 1997, 42:727-736.

27. Sureshkumar KK, Patel BM, Markatos A, et al.: Quality of life after organ transplantation in type 1 diabetics with end-stage renal disease. Clin Transplant 2006, 20:19-25.

28. Sureshkumar KK, Mubin T, Mikhael N, et al.: Assessment of quality of life after simultaneous pancreas-kidney transplantation. Am J Kidney Dis 2002, 39:1300-1306.

29. Paty BW, Lanz K, Kendall DM, et al.: Restored hypoglycemic counterregulation is stable in successful pancreas transplant recipients for up to 19 years after transplantation. Transplantation 2001, 72:1103-1107.

30. Allen RD, Al-Harbi IS, Morris JG, et al.: Diabetic neuropathy after pancreas transplantation: determinants of recovery. Transplantation 1997, 63:830-838.

31. Venstrom JM, McBride MA, Rother KI, et al.: Survival after pancreas transplantation in patients with diabetes and preserved kidney function. JAMA 2003, 290:2817-2823.

32. - Sampaio MS, Poommipanit N, Cho YW, et al.: Transplantation with pancreas after living donor kidney vs. living donor kidney alone in type 1 diabetes mellitus recipients. Clin Transplant 2009 Dec 30 [Epub ahead of print]. Recent clinical outcomes with PAK demonstrate that PAK may be similar to SPK, in that longer-term survival is enhanced by pancreas transplant despite early risks.

33. Kleinclauss F, Fauda M, Sutherland DE, et al.: Pancreas after living donor kidney transplants in diabetic patients: impact on long-term kidney graft function. Clin Transplant 2009, 23:437-446.

34. - Fridell JA, Mangus RS, Hollinger EF, et al.: The case for pancreas after kidney transplantation. Clin Transplant 2009, 23:447-453. The largest single-center comparison of SPK versus PAK demonstrates that PAK is as effective and safe as SPK.

35. Wolfe RA, McCullough KP, Schaubel DE, et al.: Calculating life years from transplant (LYFT): methods for kidney and kidneypancreas candidates. Am J Transplant 2008, 8(4 Pt 2):997-1011.

36. Wiseman AC, Wainright JL, Sleeman E, et al.: An analysis of the lack of donor pancreas utilization from younger adult organ donors. Transplantation 2010, in press.

37. Axelrod DA, Sung RS, Meyer KH, et al.: Systematic evaluation of pancreas allograft quality, outcomes and geographic variation in utilization. Am J Transplant 2010, 10:837-845. 